\title{
A NEW COOLING TECHNIQUE FOR TARGETS OPERATING UNDER VERY INTENSE BEAMS
}

\author{
FELICE IAZZI I,, SERGIO FERRERO ${ }^{1}$, RICCARDO INTROZZI $^{1,2}$, FEDERICO PINNA $^{1,2}$, \\ LUCIANO SCALTRITO ${ }^{1}$, DANIELA CALVO ${ }^{2}$, MARIA FISICHELLA $^{2}$, CLEMENTINA AGODI $^{3}$, \\ FRANCESCO CAPPUZZELLO ${ }^{3,4}$, DIANA CARBONE ${ }^{3} \&$ MANUELA CAVALLARO $^{3}$ \\ ${ }^{1}$ Department of Applied Science and Technology, Politecnico di Torino, Italy \\ ${ }^{2}$ INFN, Sezione di Torino, Italy \\ ${ }^{3}$ INFN-LNS, Catania, Italy \\ ${ }^{4}$ Department of Physics and Astronomy, Catania University, Italy
}

\begin{abstract}
The NUMEN experiment aims to study Double Charge Exchange nuclear reactions at low energy as complementary information of the Neutrino-less Double Beta Decay half-life, for the evaluation of the Majorana effective neutrino mass. Special targets of Sn, Cd, Te, Ge and Se are needed, and their thickness must be in the range between 200 and $500 \mathrm{~nm}$, in order to satisfy the energy resolution requirements of the produced ions. The very low cross-section requires high intensity of the beams; thus, the energy loss becomes a constant heat source in the target region illuminated by the beam spot. To avoid the increase of the target temperature up to the melting point, some way must be found to dissipate the heat outside the target. The solution adopted in previous experiments at much lower intensity (thin films of isotopes supported by metal frames) is not suitable in this case. Solving the heat equation in steady state condition and cylindrical coordinates gives temperatures higher than the materials melting point, due to the low internal conductivity of the targets. Therefore, we designed a new cooling technique, which takes advantage of the electron stripper located beyond the target. We changed the stripper material (usually Mylar) in pyrolytic graphite of equivalent thickness $(\approx 10 \mu \mathrm{m})$, which becomes the substrate of the isotope deposition. Due to the presence of discontinuities in the material and heat source geometry, the temperature equation has been numerically solved. Thanks to the high internal conductivity of the graphite, the results are promising. Concerning the deposition, the goal is twofold: to obtain an isotope film with uniform density, and to create a good degree of adhesion to the graphite substrate. After the first trials with Sn, heating up the substrate during the deposition seems to provide reliable and satisfactory results.
\end{abstract}

Keywords: double charge exchange, neutrino-less double beta decay, graphite substrate, thin layers, heat transfer, intense beams.

\section{INTRODUCTION}

The today investigations in the field of the Nuclear Physics often require measurements of the cross section of rare reactions. Therefore, in order to obtain a significant statistic of the data, it is necessary to use highly intense beams of charged particles, like protons or ions. These particles lose energy by ionization inside the target, generating heat and therefore increasing the target temperature. The easiest technique for cooling the target is the simple contact with a cold frame, as often used with low intensity beams and thick targets. In some recent experiments, the beam intensity increases and the targets must satisfy special requirements of thickness and composition. These requirements make the heating of the target a serious problem and pose severe constraints to the cooling system.

This is the case of the NUMEN experiment, which aims to measure some Double Charge Exchange (DCE) reactions, gathering information to better evaluate the Neutrinoless Double Beta Decay matrix elements [1], [2]. In this experiment, the targets are very thin, in order to reduce as much as possible, the probability of scattering of the reaction products, as will be shown in the next chapter. In this way, a sufficient accuracy of the energy measurement is guaranteed. 
On the other hand, the small thickness makes very difficult to build and handle such films. Moreover, the choice of the target materials depends on the reaction under study. In some cases, their thermal conduction coefficients are very low. Therefore, the design of a cooling system independent on the characteristics of the targets is mandatory.

In the following chapters, the main features of the DCE reactions and of the NUMEN experiment will be described, together with the target geometry used in previous measurements at low beam intensity. Then, the design of the new target-cooler system, satisfying the cooling requirements at high beam intensity will be illustrated. Finally, the preliminary results of the Tin depositions on Pyrolytic Graphite and the calculations of the time evolution of the temperature inside the target will be reported.

\section{DOUBLE CHARGE EXCHANGE REACTIONS IN NUMEN}

The goal of the NUMEN experiment is to investigate the nuclear matrix element of particular DCE reactions, whose initial and final states are the same as those ones of the neutrino less double beta-decay $(0 v \beta \beta)$ [3], [4]. These measurements, to be held at LNS laboratories of INFN, in Catania (Italy), will provide help in the evaluation of the nuclear matrix elements entering the expression of the lifetime of the $0 v \beta \beta$ decay.

\subsection{The DCE reactions in NUMEN}

Using the ${ }^{18} \mathrm{O}$ and ${ }^{20} \mathrm{Ne}$ ion beams of the K800 Superconducting Cyclotron (CS) and of the MAGNEX large acceptance magnetic spectrometer at LNS [5], NUMEN experiment will measure the differential cross sections of the following reactions:

1. ${ }^{116} \mathrm{Sn}\left({ }^{18} \mathrm{O},{ }^{18} \mathrm{Ne}\right){ }^{116} \mathrm{Cd}$

2. ${ }^{116} \mathrm{Cd}\left({ }^{20} \mathrm{Ne},{ }^{20} \mathrm{O}\right){ }^{116} \mathrm{Sn}$

3. ${ }^{130} \mathrm{Te}\left({ }^{20} \mathrm{Ne},{ }^{20} \mathrm{O}\right){ }^{130} \mathrm{Xe}$

4. ${ }^{76} \mathrm{Ge}\left({ }^{20} \mathrm{Ne},{ }^{20} \mathrm{O}\right){ }^{76} \mathrm{Se}$

5. ${ }^{76} \mathrm{Se}\left({ }^{18} \mathrm{O},{ }^{18} \mathrm{Ne}\right){ }^{76} \mathrm{Ge}$

The energy of the beams will range between 15 and $30 \mathrm{MeV} / \mathrm{u}$ in a first phase and up to $60 \mathrm{MeV} / \mathrm{u}$ in a second phase after the upgrading of CS. After such upgrade, the expected intensities of both beams will reach $50 \mu \mathrm{A}$, the radial profile is Gaussian, with FWHM of about $1 \mathrm{~mm}$. The materials of the five targets will be highly pure isotopes of ${ }^{116} \mathrm{Sn},{ }^{116} \mathrm{Cd}$, ${ }^{130} \mathrm{Te},{ }^{76} \mathrm{Ge},{ }^{76} \mathrm{Se}$. The first step of the design of the target setup, which include the cooling system, was dedicated to the Tin isotope ${ }^{116} \mathrm{Sn}$. As already mentioned, the thickness of the target plays a crucial role in the precision of the energy measurement. Preliminary calculations indicate that the suitable range for the Tin film is between 250 and $400 \mathrm{~nm}$.

The reaction products of DCE are ions, whose energy is measured by means of the MAGNEX spectrometer and a system of detectors [6]. In order to distinguish different isotopes, their charge must be the "full-stripped ion" charge. This requires a thin film of light material located downstream the target, whose task is to spoil the ions from the residual electrons. This film will play also a crucial role in cooling the thin target, as shown in this paper.

\subsection{Geometry of the target}

As mentioned before, the Tin target was the first to be designed. In addition to the required thickness, the other constraint on the geometry is to avoid interactions of the beam with the target frame. The chosen shape is a circular tin film with a diameter of $\approx 1 \mathrm{~cm}$. With this 
size, nearly all ions of the beam encounter the target, so that the number of the ions passing through the frame is negligible. A crown-shaped frame, clamping the external part of the target, must be maintained at constant cold temperature, as shown in Fig. 1(a).

\subsection{Heat production in the targets}

The ion beam, passing through the target, deposits energy mainly due to 3 different interactions: a) ionization, b) scattering on nuclei, c) fragmentation of nuclei. The contribution of the first process is largely dominant over the contribution of the other 2 interactions. Therefore, calculations taking into account only this contribution to the heat production are accurate enough for design purposes.

The energy lost by ionization by a single charged particle is given by the Bethe-Bloch formula [7]. The total energy lost by an ion beam is proportional to the intensity. For the intermediate energies of the NUMEN experiment, the energy loss is nearly proportional to the inverse of the ion energy. Moreover, due to the very small thickness of these targets, the energy loss rate is constant and therefore the total lost energy is proportional to the target thickness.

In Fig. 2 the total energy lost is reported as a function of the ion beam energy/nucleon, for each projectile-target couple. The beam intensity used in the calculations is $1 \mu \mathrm{A}$ and the target thickness is $1 \mu \mathrm{m}$. For other values of intensity and thickness, one has just to scale linearly the lost energy.

\subsection{Heat flow inside the targets}

Passing through the target, the beam deposits energy by ionization, acting like a uniform heat source. The shape of this source is nearly cylindrical, with a diameter of about $1 \mathrm{~mm}$, since the most part of the ions lie within the FWHM of the Gaussian distribution. The heat

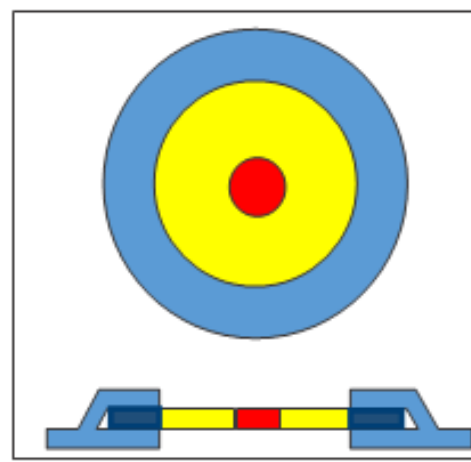

(a)

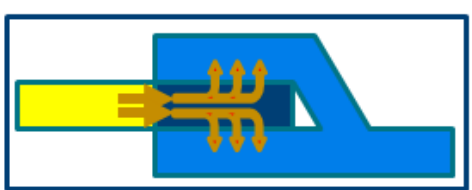

(b)

Figure 1: (a) Scheme (not in scale) of the top view (upper part) and side view (lower part) of the target. The red circle represents the region illuminated by the beam spot, the blue crown is the cooled clamped region, while the yellow part represents the region, through which the heat passes from the center to the cold frame; (b) Schematic picture of the flow of heat from the center of the target to the frame at cold temperature. 


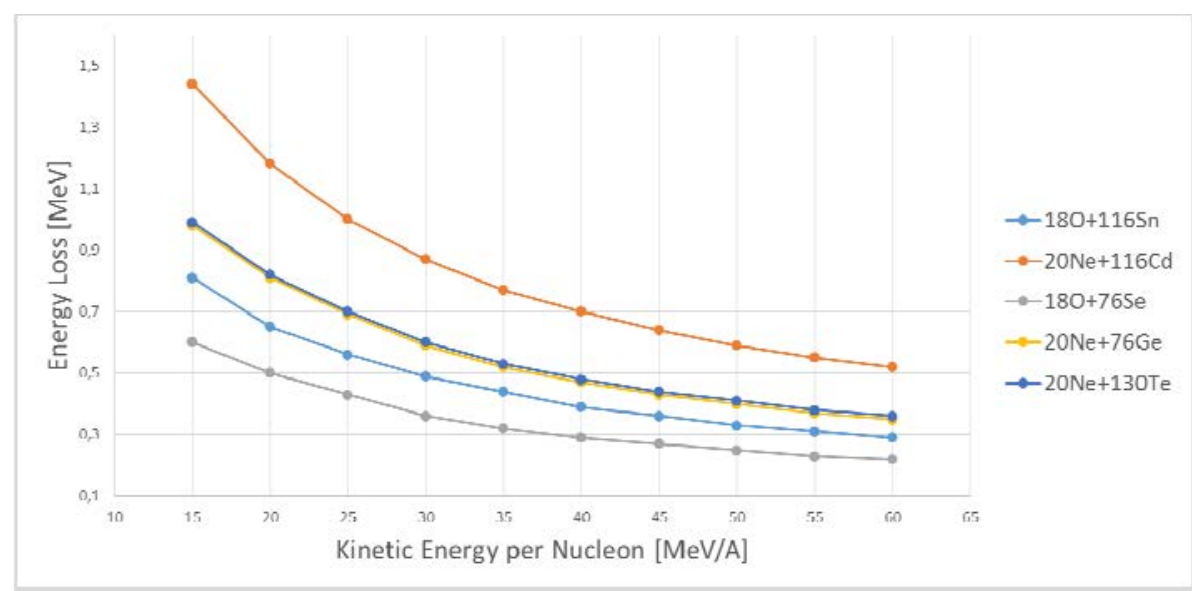

Figure 2: Total energy lost in all the reactions inside $1 \mu \mathrm{m}$ thick targets as a function of the $1 \mu \mathrm{A}$ intense ion beam energy.

flows from the center of the target to the boundaries, where it is absorbed by the cold frame, as schematically shown in Fig. 1(b). The cooling system in NUMEN will use liquid nitrogen (LN2): a pessimistic estimate of the heat losses in the refrigerator circuit leads to expect a temperature of the frame not exceeding $100 \mathrm{~K}$. In order to avoid the melting of the target in the region under the beam spot, the heat entering in the target (in the red region of Fig. 1(a)) in the unit time must be equal to the heat flowing into the cold frame (blue region in Fig. 1(b)). Such steady-state condition should be reached by the system after a transient time. After that time, the temperature remains constant and satisfies the stationary temperature equation and the Fourier Law (eqns (1) and (2)):

$$
\begin{gathered}
\frac{1}{r} \frac{d}{d r}\left(r \frac{d \Theta(r)}{d r}\right)+\frac{d Q}{k \cdot d V d t}=0 \\
\frac{d Q}{d t}=-k \cdot s \cdot 2 \pi \cdot r \cdot \frac{d \Theta(r)}{d r}
\end{gathered}
$$

Both equations (1) and (2) are expressed in cylindrical coordinates, thanks to the symmetry of the target-frame system. The parameters entering in the equations are the internal conduction coefficient $k$, the target thickness $s$, the temperature $\Theta(r)$, as a function of the radial coordinate $r$, the rate of the heat entering in the target within the beam spot, the heat rate density $\frac{d Q}{d V d t}$ of the source. Calling $\frac{d E}{d x}$ the energy loss of each ion and $I$ the beam intensity, the heat rate density is equal to the product of the intensity and the energy loss. The solution $\Theta(r)$ of Eq. (1) contains the asymptotic temperatures $\Theta_{B}$ and $\Theta_{C}$ of the spot region (radius $r_{B}$ ) and of the cold frame (radius $r_{C}$ ) respectively. Inserting $\Theta(r)$ in eqn (2), one gets:

$$
I \cdot \frac{d E}{d x}=k \cdot s \cdot 2 \pi \cdot \frac{\Theta_{B}-\Theta_{C}}{\ln \left(r_{C}\right)-\ln \left(r_{B}\right)}
$$


eqn (3) shows a linear dependence of the temperature at the beam spot radius on the beam intensity. To avoid melting of the target in the beam spot region, the temperature $\Theta_{B}$ must be lower than the melting temperature. To avoid melting of the target in the beam spot region, the temperature $\Theta_{B}$ must be lower than the melting temperature $\Theta_{M}$. By substituting $\Theta_{B}$ with $\Theta_{M}$ in eqn (3), the maximum beam intensity, which cannot be overcome without melting, is obtained.

The upper limit of the intensity is plotted in Fig. 3, as a function of the kinetic energy, for all the DCE reactions, together with the melting point of each target. It must be remarked that: a) no beam intensity exceeding $2.5 \mu \mathrm{A}$ is allowed at the lowest energy, b) also at higher energy the beam intensity should remain below $2.5 \mu \mathrm{A}$. Taking into account that all the DCE reactions must be investigated in a range between 15 and $60 \mathrm{MeV} / \mathrm{A}$ and that the beam intensity is needed to reach up to $50 \mu \mathrm{A}$, it is evident that the above described target-cooler system is not sufficient for the purposes of the NUMEN measurements. A different setup for Tin target and cooler has been investigated, as described in the next chapter.

\section{THE TARGET-COOLER SYSTEM IN NUMEN}

The high beam intensities, required in the planned investigations of the DCE reactions, require a more powerful cooling technique than that one described in the previous chapter and often used with low intensity beams. The internal conductivity plays a crucial role in the dissipation of the heat from the center of the target to the cold region and from eqn (3) it can be inferred that greater is the conductivity, higher is the allowed beam intensity. Unfortunately, the choice of the target materials depends on the reactions under study. A way to accelerate the heat transfer is to deposit the target material on a substrate, to which

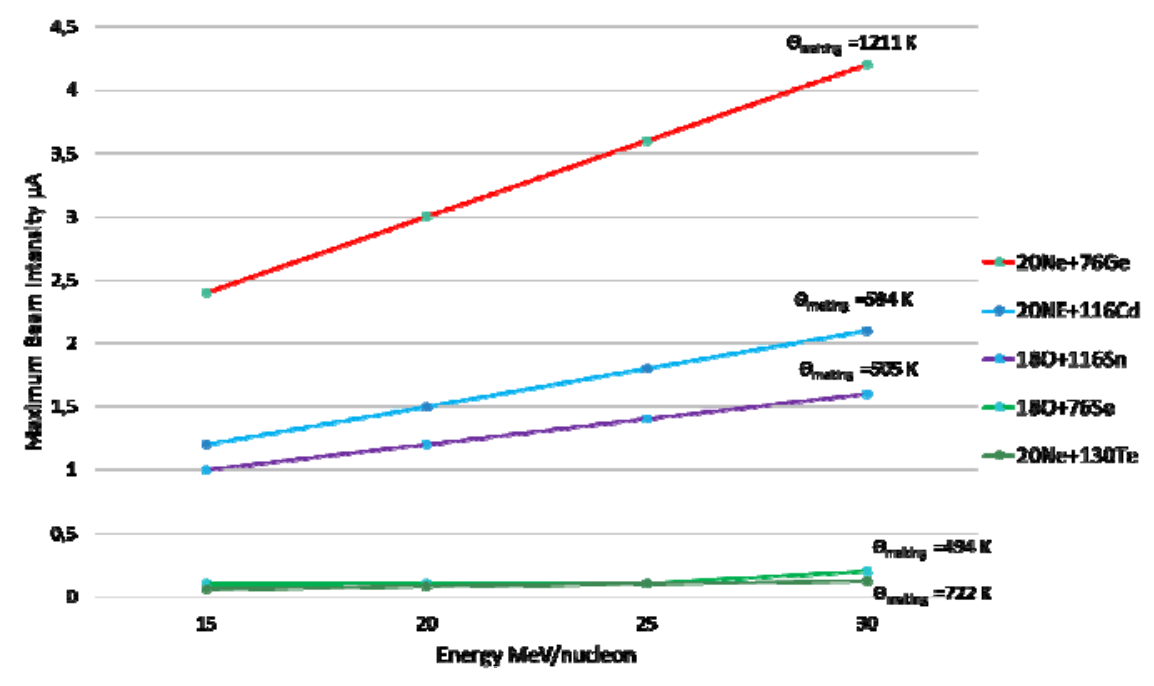

Figure 3: Upper limit of the beam intensity as a function of the energy of the projectile ion. The melting temperature of the targets is indicated near the line of each DCE reaction. 
the heat can rapidly flow to the cold frame. This substrate must satisfy several constraints and the cooling efficiency has to be evaluated: the results are reported in the next paragraphs.

\subsection{Deposition of target on a substrate}

A target made by a deposited material onto a substrate presents some problems from the point of view of the detection of the reaction products. In fact, the accuracy of the energy measurement of the ions exiting from the reaction is affected by the energy loss and by eventual nuclear scattering in the substrate, if it is located downstream the beam. Spurious products can exit from the interaction of the beam with the substrate but they can be rejected by the particle identification procedures. On the other hand, if the substrate is located upstream, the spatial shape and the energy distribution of the beam are spread, increasing the errors.

As mentioned in Section 2.1, the DCE measurements require an electron stripper to spoil the ion products from the residual electrons. In previous measurements [8] at low beam intensity and lower energy, a thin Mylar film, $\approx 7 \mu \mathrm{m}$ thick, served this purpose. In the NUMEN experiment the substrate, located downward, plays this role.

The pyrolytic graphite has been chosen as substrate, due to the very high thermal conductivity. This material is basically a stack of several graphene sheets, is flexible and mechanically resistant and has a very high thermal conductivity in the sheet plane, through which the heat can flow from the beam spot to the cold frame.

The first deposition was made using natural Tin and the adhesion was satisfying. The structure of the deposited film on graphite showed a granularity with sizes up to $500 \mathrm{~nm}$, which affects the evaluation of the loss energy of the products. Fig. 4 shows the FESEM microscopy of the cross section of the Tin deposition. The nominal thickness is $500 \mathrm{~nm}$ but some agglomerates reach sizes of $\approx 1 \mu \mathrm{m}$. The use of some techniques (annealing at different temperatures, deposition on hot substrate) was successful in obtaining a uniform structure, as described in [8].

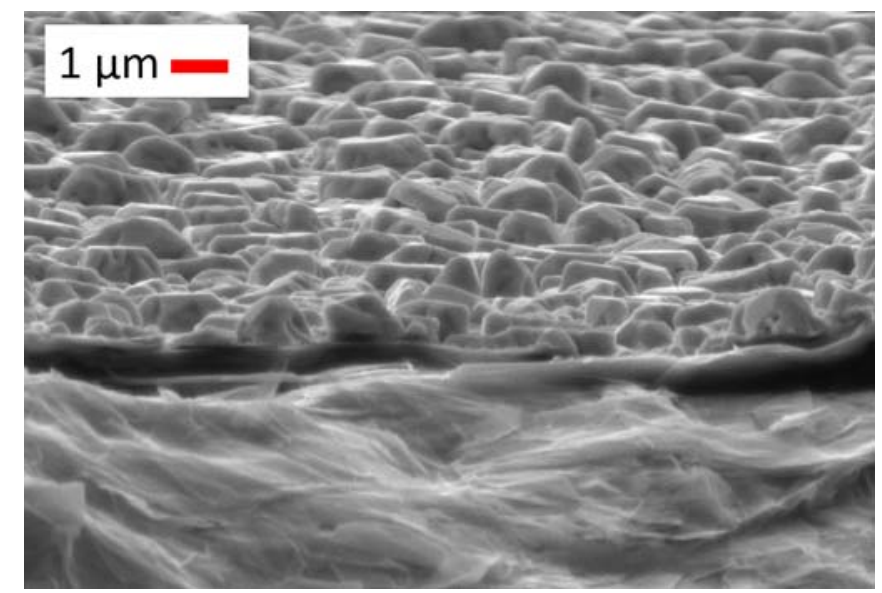

Figure 4: FESEM microscopy of the deposition of $\mathrm{Sn}$ on a graphite substrate: the picture shows a cross section of the deposition, with substrate in the lower part and $\mathrm{Sn}$ in the upper part. In the upper part of the figure, one can notice the high bumps and clusters of the Sn film. 


\subsection{Geometry of the target-substrate system}

The new geometry of the system target-substrate is schematized in Fig. 5. The green disk represents the Tin deposition, the grey disk is the graphite right below the target and the teal annulus represent the graphite clamped by the cold frame at fixed temperature $\Theta_{\mathrm{C}}$. The beam passes through both Tin and Graphite with a spot of $1 \mathrm{~mm}$ in diameter, in the direction of the $\mathrm{z}$-axis. The thickness of $\mathrm{Sn}$ is $t_{T} \approx 500 \mathrm{~nm}$ and that one of graphite is $t_{G} \approx 10 \mu \mathrm{m}$.

In this configuration, the heat generated by the beam at the center of the target and graphite disks flows toward the colder regions, as shown in Fig. 6. In particular, the red zone (target part illuminated by the beam) and the orange zone (substrate part illuminated by the beam) are sources of heat. They are different because of the different energy loss in both materials. The green and grey regions (not illuminated target and substrate) are colder, hence they receive heat from the center and transmit it to the cooled regions (clamped substrate and frame). Since the internal conductivity of the Graphite is much higher than the one of the Tin, the temperature in the grey zone is expected to be lower than in the green zone. Therefore, part of the heat in Tin flows also toward the substrate. The evaluation of these flows and temperatures is reported in the next chapter.

\section{THE SPATIAL AND TIME EVOLUTION OF THE TEMPERATURE}

Before switching on the beam, the initial temperature in the whole target-substrate-frame system is given everywhere by the temperature of the cooling system.

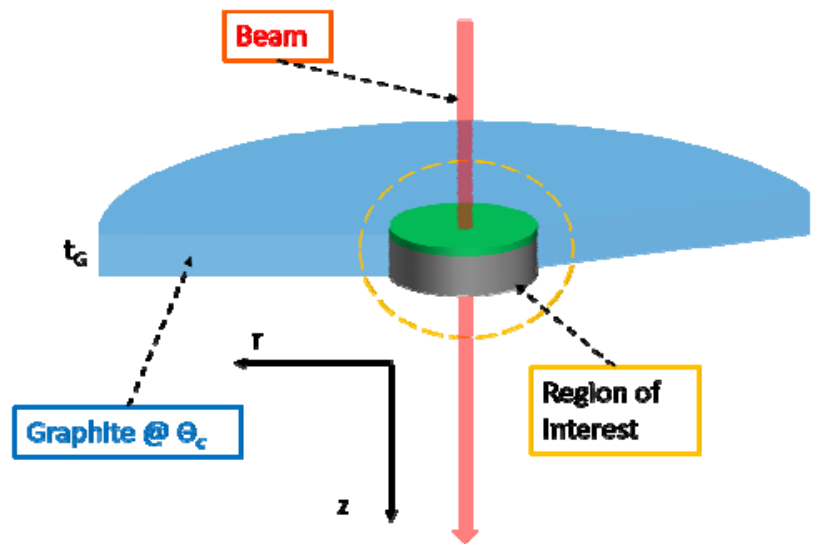

Figure 5: Sketch (not to scale) of the target-substrate system.

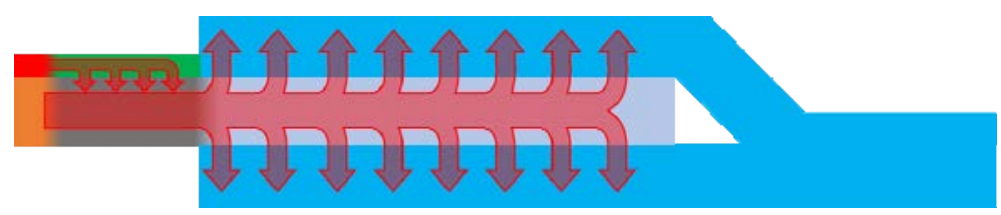

Figure 6: Scheme of the heat flow from the hot regions to the cold substrate clamped by the cold frame. 
By using a $\mathrm{LN}_{2}$-circuit to cool down the frame, a temperature not higher than $100 \mathrm{~K}$ is easily reachable. The illuminated region can be approximated by a hot cylinder of radius $0.5 \mathrm{~mm}$ for the reasons explained in Section 2.4. Therefore, a discontinuity along the radius of the heat source is created in the boundary of this cylinder. As mentioned before, the cylinder has different heat rates in target and in substrate and this creates another discontinuity of the heat source along the z-axis. Further discontinuities are present in the contact surfaces between target and substrate (green and grey zones in Fig. 6) and between them and the cold region (blue). The presence of such discontinuities suggests evaluating the evolution of the temperature in all points of the target-substrate system using a numerical approach, as shown in the next Section.

\subsection{Numerical solution of the heat equation}

The transfer of heat to the cold frame modifies, along the time, the temperature inside the target and substrate, satisfying the not-stationary heat equation (in cylindrical coordinates):

$$
\frac{k_{r}}{\rho \cdot c} \cdot\left(\frac{\partial^{2} \Theta}{\partial r^{2}}+\frac{1}{r} \frac{\partial \Theta}{\partial r}\right)+\frac{k_{z}}{\rho \cdot c} \cdot \frac{\partial^{2} \Theta}{\partial z^{2}}+\frac{1}{\rho \cdot c} \cdot \frac{d Q}{d V \cdot d t}=\frac{\partial \Theta}{\partial t}
$$

The Sn has an isotropic thermal conductivity, hence in eqn (4) the thermal conductivity coefficients $k_{r}$ (along the radius) and $k_{z}$ (along z-axis) are the same, while they are different in the Pyrolytic Graphite. The density and the specific heat are homogeneous in each material. The heat source $\frac{d Q}{d V \cdot d t}$ is equal to the total heat produced in the unit time by the beam in Tin and Graphite, divided by the volumes of the illuminated cylinders. The intensity of the ${ }^{16} \mathrm{O}$ beam used in these calculations was $50 \mu \mathrm{A}$. The bi-dimensional grid along the radius and along the z-axis has been chosen in such a way that no cell lays across the discontinuity surfaces. The variables $r$ and $z$ in eqn (4) have been modified to include the coefficients of the derivatives. The evolution in time has been calculated starting from the initial, uniform cold temperature. For the calculation of the derivatives the "central difference" formula has been applied. The results are reported in the next Section.

\subsection{Results of the numerical calculations}

The solution of eqn (4) gives the value of the temperature in each point of coordinates $(r$, $z$ ), at different times. The spatial distribution of the temperature (in Kelvin) has been calculated every $\Delta t \approx 3.7 \cdot 10^{-10} \mathrm{~s}$ and is reported in two-dimensional plots of the same type of Fig. 7, where the $\mathrm{z}$ coordinate is named Depth and the $\mathrm{r}$ coordinate is named Radius.
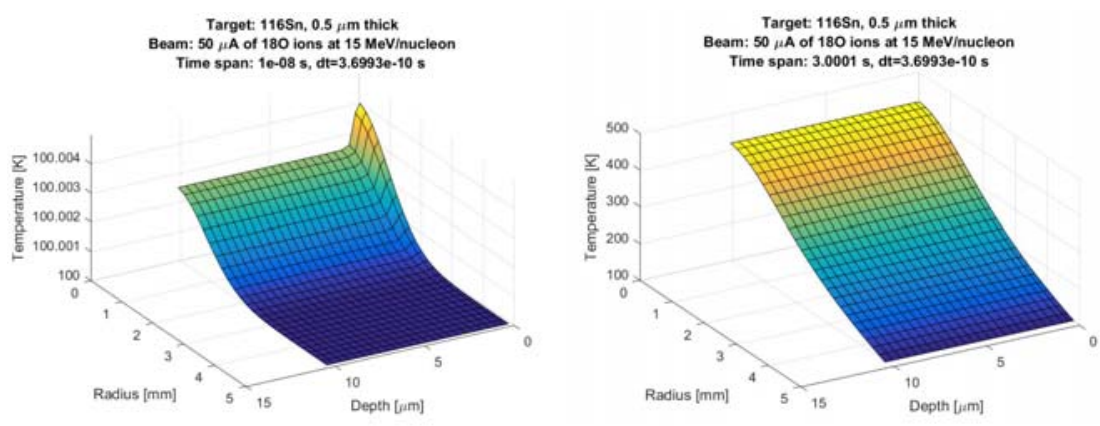

Figure 7: Spatial temperature distribution: after 10ns (left), after 3s (right). 
The plot on the left in Fig. 7 represents the distribution after 10ns: after such a short time only the central zone of Tin, which is under the beam spot, is (slightly) warmed, while the rest of the space is still cold. The plot on the right shows the evolution after $3 \mathrm{~s}$ : here the temperature is quite uniform along $\mathrm{z}$, proving that the heat flows abundantly and rapidly from Tin to Graphite. From Graphite, heat flows quickly to the cold frame. The intermediate plots, not reported here, show that: a) the maximum temperature is always in the point $r=0, z=0$, i.e. in the center of the beam spot, as expected; $b$ ) after $0.1 \mathrm{~s}$ this value does not change appreciably, i.e. the temperature inside the target and substrate reached the asymptotic maximum value.

Since the maximum temperature in the Tin target, reported in Fig. 7 (right), is less than $450 \mathrm{~K}$, largely below the melting point of the Tin $(505 \mathrm{~K})$, these calculations prove that the Pyrolytic Graphite can increase the transfer of heat from the Tin target to the cold frame.

\section{CONCLUSIONS AND FUTURE WORK}

The use of intense ion beam at intermediate energy requires a special attention to the problem of the cooling of the targets, whose temperature can reach values higher than the melting point, as in the case of the NUMEN experiment. The high intensity produces a large amount of heat inside the target and an efficient cooling system is necessary. On the other hand, the needs of high statistics make unavoidable this option. The nature of the targets, in general heavy isotopes, produces a large amount of heat, which does not transfer rapidly to the cold frame, due to the low internal thermal conductivity of such isotopes.

The present work deals with the problem of the cooling, designing a system of target and substrate: the target (Tin) is deposited on a Pyrolytic Graphite substrate, whose thermal conductivity in the plane of the surface is very high. The presence, in this kind of experiments, of a thin film of light elements (typically carbon light compounds), as stripper of the residual electrons of the produced ions, suggested the idea of a double layer targetsubstrate.

The first problem in making such system was the adhesion of Tin on Pyrolytic Graphite and the structure of the deposition. After some trials, a satisfying result was obtained. Then, the efficiency of the cooling by means of the substrate has been evaluated, solving the heat equation by numerical methods. The result is positive because the maximum temperature reached by the target is largely below the melting point.

The future work foresees some further tests of the efficiency of the cooling and the design of the targets made by the other nuclei, listed in Section 2.1.

A preliminary test will be done using a LASER beam to heat a spot of the Tin-Graphite system, with the frame at room temperature. The temperature of both target and substrate will be measured and the result will be compared with the predictions of dedicated numerical calculations. If positive, the assumption of good contact between target and substrate, made in the calculations, will be validated. An analogous test using an intense ion beam with the $\mathrm{LN}_{2}$ cooler will be the definite check of this cooling method.

Finally, the deposition technique will be applied to the targets of $\mathrm{Cd}, \mathrm{Se}, \mathrm{Te}$ and $\mathrm{Ge}$. Analogous numerical calculations will be performed, followed by similar experimental tests with LASER and ion beams.

\section{REFERENCES}

[1] Cappuzzello, F. et al., Eur. Phys. J. A, 51(145), 2015.

[2] Cappuzzello, F. et al., J. Phys.: Conf. Ser. 630, 2015. 12018.

[3] Agodi, C. et al., Nuclear and Particle Physics Proceedings, pp. 265-266, (28-30), 2015. 
70 Materials and Contact Characterisation VIII

[4] Cappuzzello, F. et al., The nuclear matrix elements of $0 v \beta \beta$ decay and the NUMEN project at INFN-LNS. EPJ Web of Conferences, 117, 2016. 10003.

[5] Cappuzzello, F., Agodi, C., Carbone, D., \& Cavallaro, M., Eur. Phys. J. A, 52(167), 2016.

[6] Cavallaro, M. et al., Eur. Phys. J. A, 48(59), 2012.

[7] Olive, K. et al., Chin. Phys. C 38, 2014. 090001.

[8] Pinna, F. et al., Thin Target Fabrication for the NUMEN Experiment. SurfCoat 2017 Conference Proceedings. 